\title{
The Influence of End of Day Silicone Hydrogel Daily Disposable Contact Lens Fit on Ocular Comfort, Physiology and Lens Wettability
}

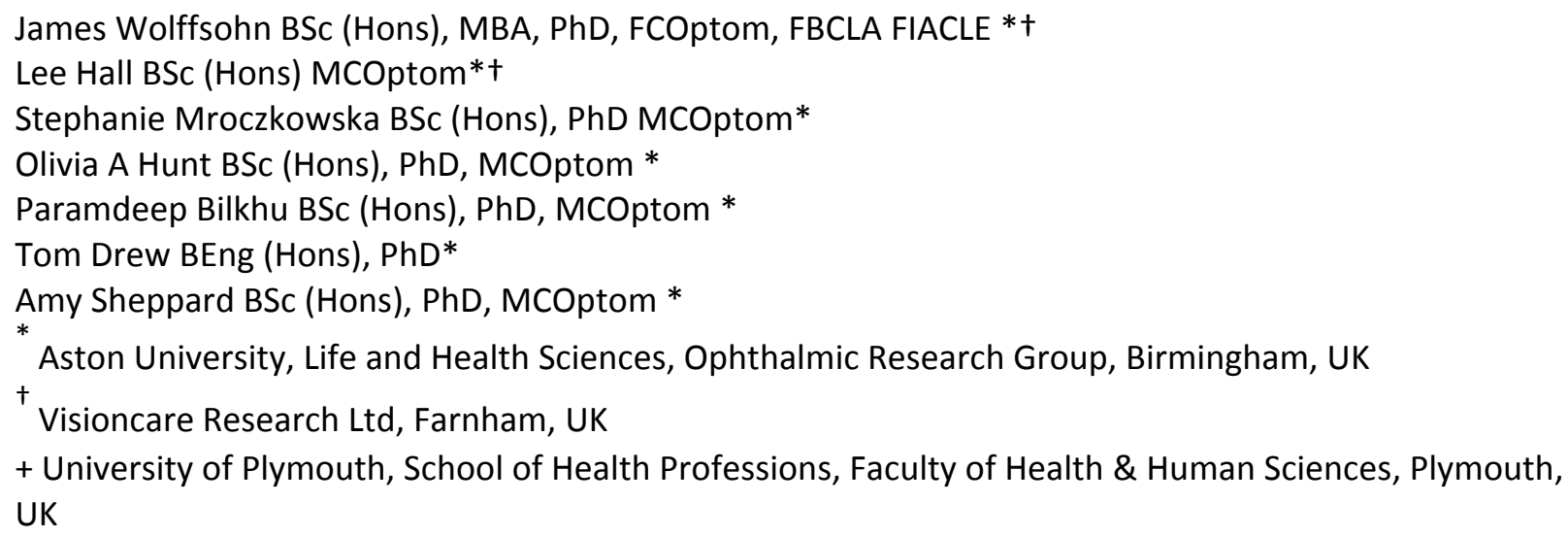




\section{ABSTRACT}

Purpose: To quantify the end-of-day silicone-hydrogel daily disposable contact lens fit and its influence of on ocular comfort, physiology and lens wettability.

Methods: Thirty-nine subjects $(22.1 \pm 3.5$ years) were randomised to wear each of 3 siliconehydrogel daily-disposable contact lenses (narafilcon A, delefilcon A and filcon II 3), bilaterally, for one week. Lens fit was assessed objectively using a digital video slit-lamp at 8, 12 and 16 hours after lens insertion. Hyperaemia, non-invasive tear break-up time, tear meniscus height and comfort were also evaluated at these timepoints, while corneal and conjunctival staining were assessed on lens removal.

Results: Lens fit assessments were not different between brands $(p>0.05)$, with the exception of the movement at blink where narafilcon A was more mobile. Overall, lag reduced but push-up speed increased from 8 to 12 hours $(p<0.05)$, but remained stable from 12 to 16 hours $(p>0.05)$. Movement-on-blink was unaffected by wear-time $(F=0.403, p=0.670)$. A more mobile lens fit with one brand did not indicate that person would have a more mobile fit with another brand $(r=-0.06-$ $0.63)$. Lens fit was not correlated with comfort, ocular physiology or lens wettability $(p>0.01)$.

Conclusions: Among the lenses tested, objective lens fit changed between 8 hours and 12 hours of lens wear. The weak correlation in individual lens fit between brands indicates that fit is dependent on more than ocular shape. Consequently, substitution of a different lens brand with similar parameters will not necessarily provide comparable lens fit.

Keywords: Contact lenses; Daily Disposables; Silicone-Hydrogel; Objective Lens fit; Lens substitution 


\section{INTRODUCTION}

The assessment of soft contact lens fit is a critical part of the prescribing process. Well fitting lenses are an essential requirement to ensure good comfort, stable vision and minimal effect on ocular integrity. However, the assessment of lens fit in clinical practice is typically made only after a few minutes on initial trial and at aftercare appointments, taking place, at most, after a working day. The average duration of contact lens wear, though, has been reported to be around $13-14$ hours a day, ${ }^{1-3}$ with approximately $25 \%$ of wearers reporting wearing their contact lenses for 16 hours, and about $6 \%$ wearing their lenses for 17 hours a day or more. ${ }^{1}$

Furthermore, patients generally report comfortable wearing times of about 1 to 1.5 hours less than their total wearing time and this appears to be a factor influencing overall wearing time in a proportion of contact lens wearers. ${ }^{1,3}$ Consequently, there is a need to understand the changes occurring in the lens-eye relationship towards the end of the wearing day. Despite this, few published studies have examined contact lens wearers who had been wearing their lenses greater than 12 hours, $^{4-7}$ and none of these evaluated silicone hydrogel daily disposable contact lenses.

Discomfort, particularly towards the end of the day, is a major cause of contact lens discontinuation. ${ }^{8}$ Dry eye symptoms are the most common complaint, ${ }^{1,4}$ with over $70 \%$ of wearers reporting symptoms late in the day, ${ }^{2}$ and approximately one-third of these discontinuing lens wear as a result. ${ }^{8}$ However, the relationship of discomfort with respect to changes in lens fit towards the end of the day has not been documented.

Silicone hydrogel contact lenses afford greater oxygen transmissibility, which result in less compromise in anterior eye physiology. However, no marked benefit in ocular comfort has been reported with these compared to traditional hydrogel lenses. ${ }^{9-11}$ Since, it is known that some combinations of contact lenses and multipurpose lens care solutions result in solution-induced corneal staining, potentially having an impact on comfort, ${ }^{12-15}$ use of the daily disposable modality eliminates this confounding effect. 
The purpose of this study, therefore, was to assess the influence of end of day fitting characteristics of silicone-hydrogel daily disposables on ocular comfort, physiology and lens wettability. 


\section{METHODS}

Thirty-nine subjects (average age $22.1 \pm 3.5$ years; $54 \%$ female) were enrolled in a randomised crossover evaluation of three silicone hydrogel daily disposable contact lenses after one week of bilateral wear. None of the subjects were on ocular medication, had incurred ocular injury or surgery within twelve weeks prior to commencing the study, had pre-existing ocular irritation or displayed evidence of systemic or ocular abnormality, infection or disease likely to affect successful wear of contact lenses. The subjects were all existing adapted spherical soft contact lens wearers and were fitted with the same power of contact lens for all three silicone hydrogels (average $-2.8 \pm 1.9 \mathrm{D}$, range -0.5 to $-7.0 \mathrm{D}$ ). The investigators were masked throughout the study, but due to the loss of sterility that would result in re-packaging, the study was open label. Subjects were, however, masked to the sponsor of the study. Subjects gave written informed consent after explanation of study procedures. The study was approved by the Aston University Human Sciences Ethical Committee and conformed to the Declaration of Helsinki.

The three silicone hydrogel daily disposable lenses used in the study were those currently marketed in the UK at the time the study was conducted: 1-DAY ACUVUE ${ }^{\circledR}$ TruEye $^{\circledR}$ (Vistakon, Johnson and Johnson, Jacksonville, Florida, USA), Clariti ${ }^{\mathrm{TM}} 1$ day (Sauflon Pharmaceuticals, London, UK) and DAILIES TOTAL $1^{\circledR}$ (Alcon, Fort Worth, Texas, USA) (Table 1).

Measures were taken at three time points throughout the final day of wear for each lens type, at 8, 12 and 16 hours after lens insertion. The assessment of dynamic lens fit was captured using a digital slit lamp (CSO, Scandicci, Italy) with a digital camera of resolution 1392 x 1024 pixels, frame rate $11 \mathrm{~Hz}$. The resulting video was analysed by a masked observer using a purposedeveloped image analysis program (LabVIEW, National Instruments, Austin, Texas, USA). Movement on blink in upgaze was assessed by the change in vertical lens position relative to the cornea from the first video frame following the blink. Lag was assessed as the difference between the limbus to lens edge distance in each of the horizontal positions of gaze compared to the same distances when viewing in primary gaze. Finally, push-up recovery speed was calculated from the change in vertical lens position relative to the cornea from the first video frame following the lens release, divided by the number of frames over which the movement 
occurred, times the frame rate. These objective measures have been previously shown to be most appropriate to define soft contact lens fit and highly repeatable. ${ }^{16}$

Table 1: Study Lens Details.

\begin{tabular}{|c|c|c|c|}
\hline Lens Type & $\begin{array}{c}1 \text { Day Acuvue } \\
\text { TruEye }^{\circledR}\end{array}$ & $\begin{array}{l}\text { DAILIES } \\
\text { TOTAL } 1^{\circledR}\end{array}$ & $\begin{array}{l}\text { Clariti }{ }^{\mathrm{TM}} \\
1 \text { Day }\end{array}$ \\
\hline Manufacturer & $\begin{array}{c}\text { Johnson \& Johnson } \\
\text { Vision Care, Inc }\end{array}$ & Alcon Ciba & $\begin{array}{c}\text { Sauflon } \\
\text { Pharmaceuticals Ltd }\end{array}$ \\
\hline Material & narafilcon $\mathrm{A}$ & delefilcon A & filcon II 3 \\
\hline Water Content (\%) & 46 & $\begin{array}{c}\sim 33 \text { at core } \\
>80 \text { at surface }\end{array}$ & 56 \\
\hline Base curve $(\mathrm{mm})$ & 8.5 & 8.5 & 8.6 \\
\hline Diameter (mm) & 14.2 & 14.1 & 14.1 \\
\hline $\begin{array}{l}\text { Oxygen Transmissibility } \\
@-3 D(D K / t)\end{array}$ & 118 & 156 & 86 \\
\hline Modulus (MPa) & 0.7 & 0.7 & 0.5 \\
\hline Storage Solution & $\begin{array}{c}\text { Buffered saline with } \\
\text { HydraClear }\end{array}$ & $\begin{array}{l}\text { Buffered saline with } \\
\text { polymeric wetting } \\
\text { agents }\end{array}$ & Buffered saline \\
\hline
\end{tabular}

Comfort was assessed subjectively on a scale from 1 to 10 (1=poor, 10=excellent). Subjective grading of bulbar and limbal hyperaemia was assessed by the same experienced investigator to one decimal place using the Efron grading scale due to its linearity. ${ }^{17}$ At the 16 hour visit, immediately after lens removal, sodium fluorescein (1mg Bausch \& Lomb, Rochester, NY, USA) and lissamine green (GreenGlo, HUB Pharmaceuticals, Rancho Cucamonga, California, USA) were instilled and any observed staining recorded as trace, mild, moderate or severe.

Non-invasive tear break-up time (NITBUT) was evaluated using a modified CA-1000 topographer (Topcon, Newbury, UK), which projected circular mires onto the corneal surface, with the tear film reflection observed on a 30 inch flat panel monitor and the NITBUT recorded at the first sign of mire distortion. An average of 3 measures was taken. Tear-meniscus height was captured with the digital slit lamp and LabVIEW programming was used to measure the average meniscus heights from the line of reflection along the top of the tear prism, to the very first visible edge of the eyelid. This technique has previously been shown to be highly repeatable. ${ }^{5}$ 


\section{Statistical Analysis}

As the data was normally distributed, repeated measures analysis of variance (ANOVA) was used to assess the differences in lens fit with the time of day and between the lens designs. Eyes were treated as repeated measures to prevent statistical bias. Comfort, hyperaemia grading, staining and tear film metrics for the right eye only were correlated against lens fit using Spearman's ranked correlation to determine whether lens performance was related to the lens design or individual characteristics. A $P$-value of 0.05 was taken to indicate significance throughout as the use of ANOVAs minimised the number of comparisons, except for correlations where a value of $P \leq 0.01$ to reduce the risk of type I errors associated with multiple comparisons of association. 


\section{RESULTS}

The lenses were worn on average $6.9 \pm 0.3$ days a week for $10.8 \pm 2.0$ hours a day, and for 16 hours on the assessment days.

\section{$\underline{\text { Lens Fit }}$}

Movement on blink ranged from 0.06 to $1.73 \mathrm{~mm}$. On blink, there was no difference with time after insertion ( 8 hours: $0.34 \pm 0.24 \mathrm{~mm} ; 12$ hours: $0.35 \pm 0.28 \mathrm{~mm} ; 16$ hours: $0.36 \pm 0.28 \mathrm{~mm} \mathrm{~F}$ $=0.403, P=0.670)$. The narafilcon A lenses moved further on blink than the other lens brands (delefilcon A: $0.33 \pm 0.21 \mathrm{~mm}$; narafilcon A: $0.41 \pm 0.34 \mathrm{~mm}$; filcon II 3: $0.33 \pm 0.25 \mathrm{~mm}$; F = 3.217, $P=0.046)$. There was no interaction between lens brands and time after insertion $(F=$ $0.423, P=0.792)$. Movement on blink with the delefilcon A lenses was significantly correlated with the narafilcon A lenses, but only after 8 hours of wear $(r=+0.527, P<0.01)$. Movement on blink was not correlated between assessment times with any of the lens brands ( $r=-0.06$ to 0.53; Table 2).

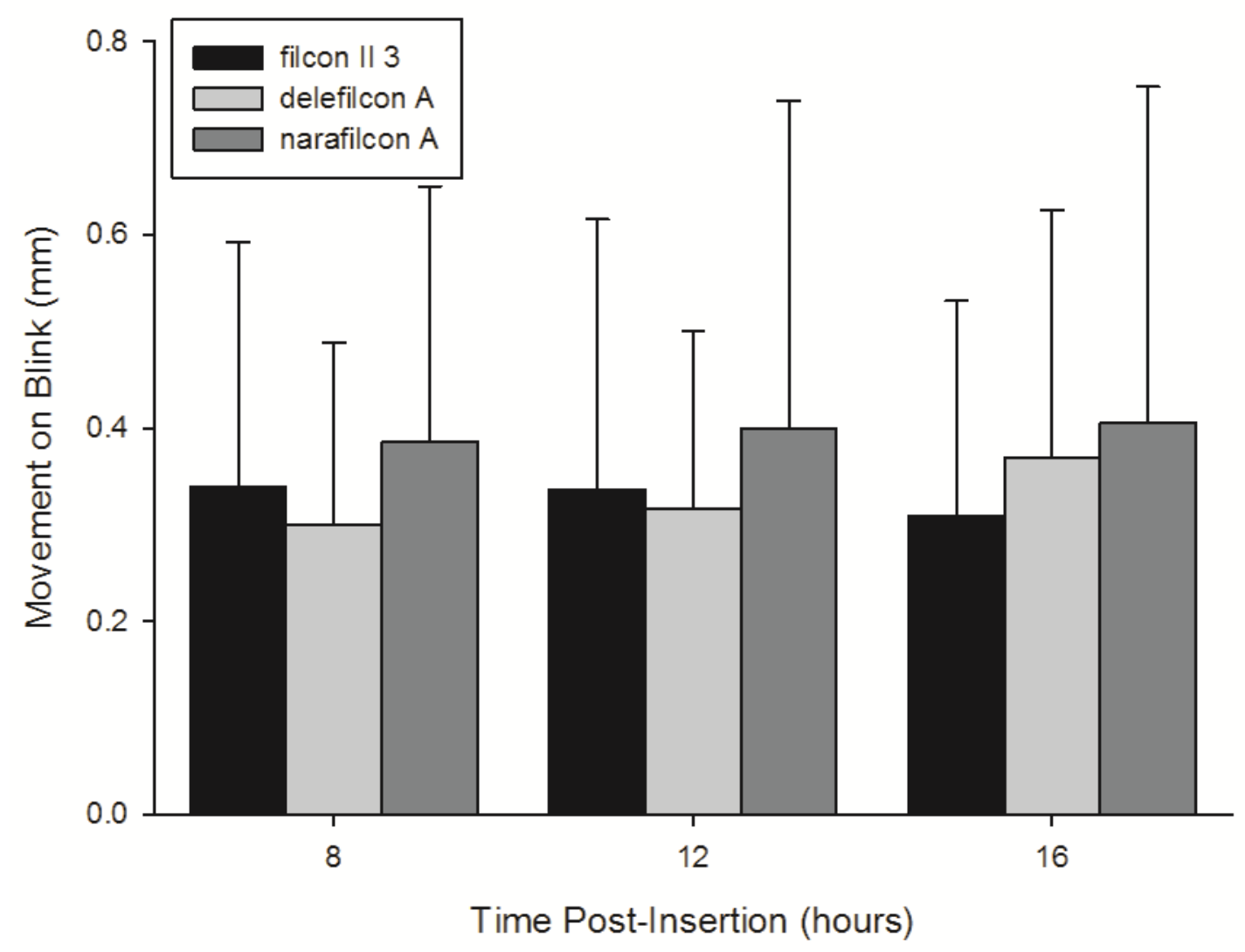


Figure 1: Movement on blink between study lenses with time after insertion. $N=39$. Error

$$
\text { bars }=1 \text { S.D. }
$$

Lag on horizontal excursions ranged $-7 \%$ to $215 \%$. Lag reduced towards the end of the day (8 hours: $77.3 \pm 52.3 \%$; 12 hours: $69.2 \pm 31.1 \%$; 16 hours: $70.1 \pm 36.5 \% ; F=3.220, P=0.046$ ). The lens brands had a similar lag (delefilcon A: $71.2 \pm 36.5 \%$; narafilcon A: $77.7 \pm 49.2 \%$; filcon II 3: $68.3 \pm 28.8 \% ; F=2.384, P=0.100)$ and there was no interaction between lens brands and time after insertion $(F=1.421, P=0.230)$. Lag was correlated for each brand between the assessment times $(r=+0.527, P<0.01)$ but generally not significantly correlated between the lens brands ( $r=0.06$ to 0.63 ; Table 2$)$.

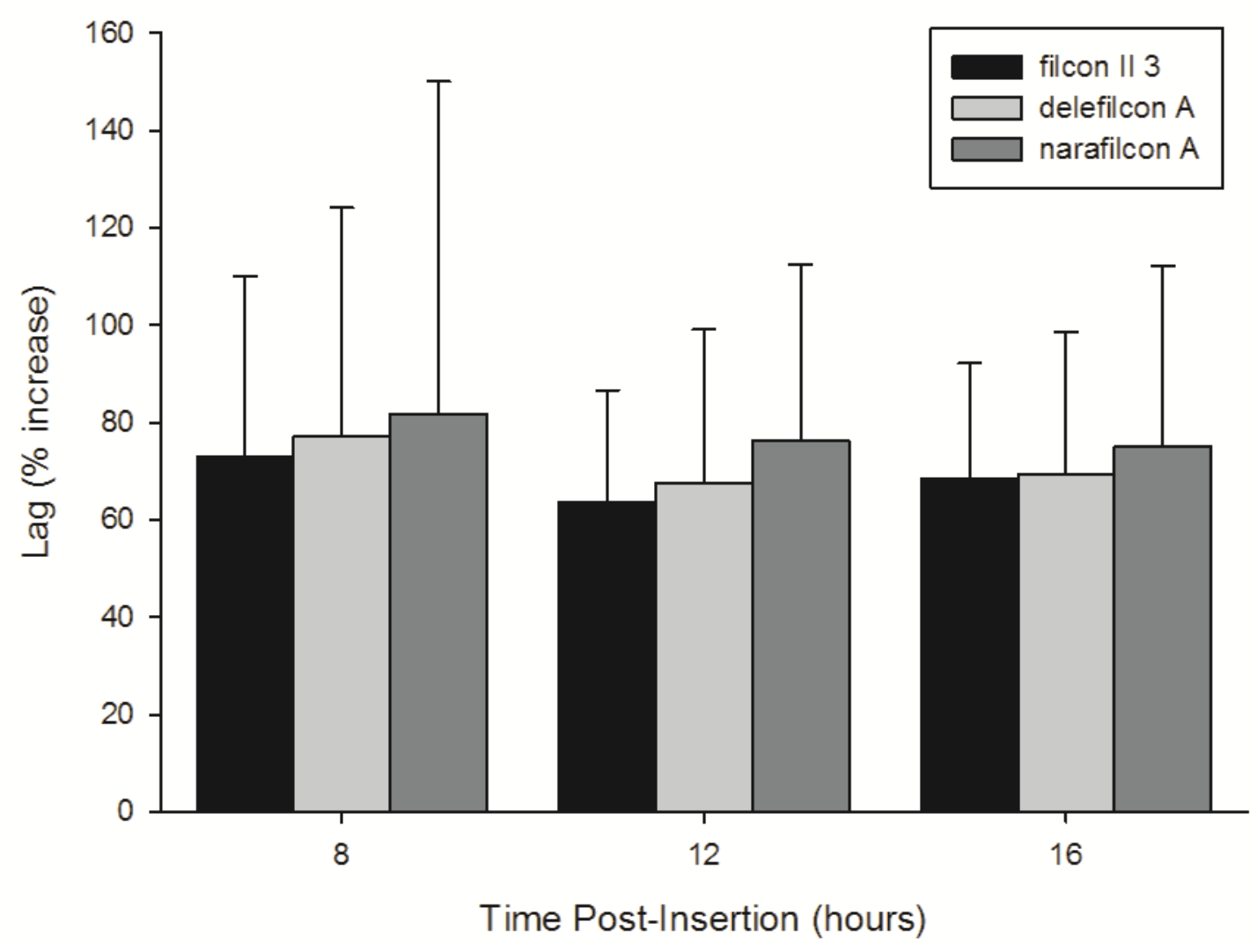

Figure 2: Increase in lag between study lenses with time after insertion. $\mathrm{N}=39$. Error bars = 1 S.D. 
Lens push-up recovery speed ranged from 0.0 to $3.4 \mathrm{~mm} / \mathrm{s}$. The lenses had a faster recovery speed after either 12 hours $(0.76 \pm 0.44 \mathrm{~mm} / \mathrm{s})$ or 16 hours $(0.73 \pm 0.40 \mathrm{~mm} / \mathrm{s})$ of wear compared to 8 hours $(0.61 \pm 0.41 \mathrm{~mm} / \mathrm{s} ; \mathrm{F}=3.345, P=0.041)$. However, the recovery speed following push-up was similar between lens brand (delefilcon A: $0.60 \pm 0.44 \mathrm{~mm} / \mathrm{s}$; narafilcon A: $0.71 \pm 0.38 \mathrm{~mm} / \mathrm{s} ;$ filcon II $3: 0.78 \pm 0.47 \mathrm{~mm} / \mathrm{s} ; \mathrm{F}=2.903, P=0.062)$, and there was no interaction between brand and time $(F=0.645, P=0.631)$. Push-up recovery speed was not correlated between the lens brands or for each brand between assessment times $(r=-0.16$ to 0.20; Table 2).

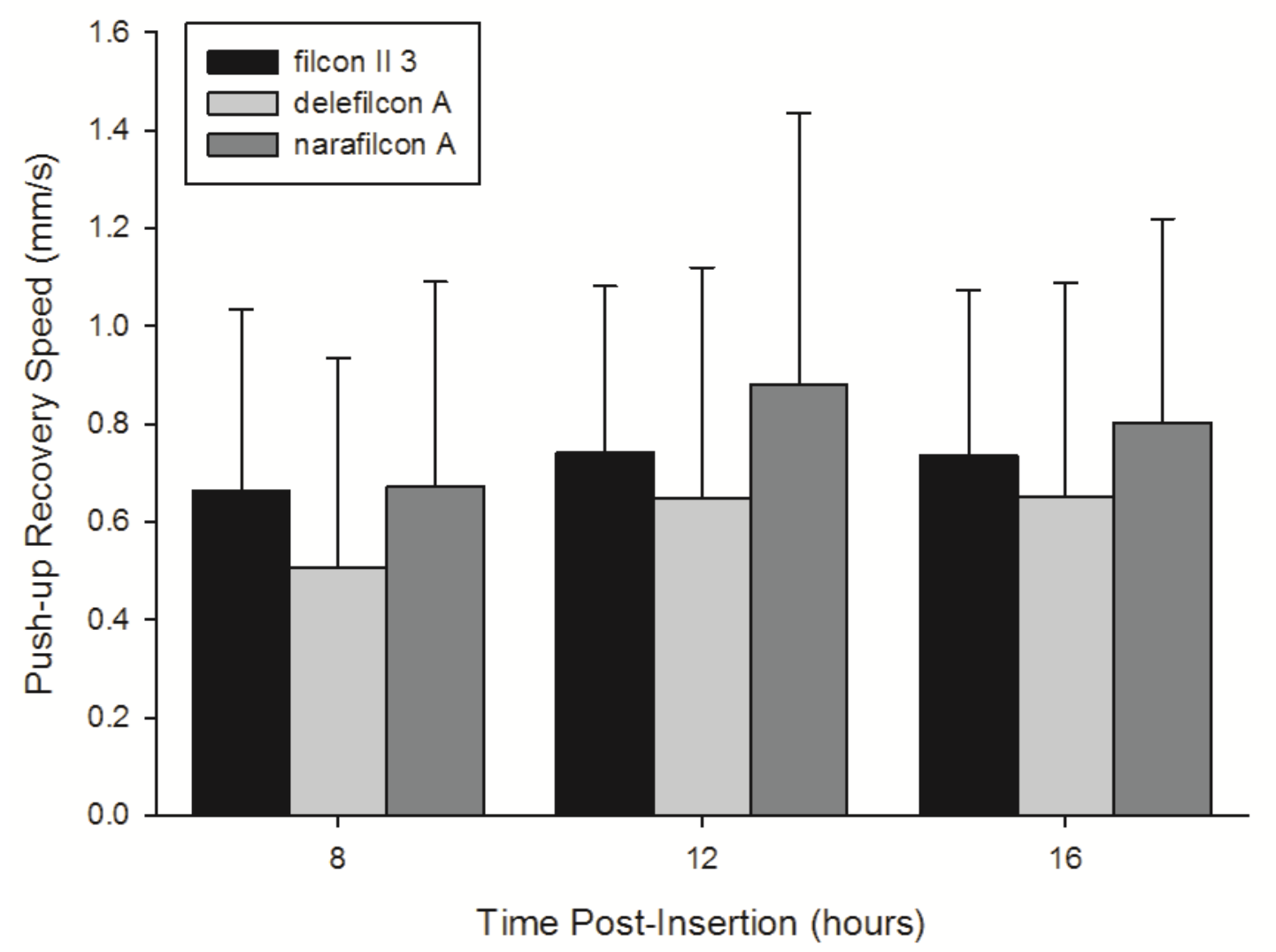

Figure 3: Push-up recovery speed between study lenses with time after insertion. N=39.

Error bars $=1$ S.D. 
Table 2: Relationship in fit variables between lens brands with time $\quad(n=39) \quad * P<0.01 ; * * P<0.001$

\begin{tabular}{|c|c|c|c|c|c|c|c|c|c|}
\hline & \multicolumn{3}{|c|}{ Blink } & \multicolumn{3}{|c|}{ Lag } & \multicolumn{3}{|c|}{ Push-Up } \\
\hline Time & $\begin{array}{l}\text { delefilcon vs. } \\
\text { narafilcon }\end{array}$ & $\begin{array}{l}\text { narafilcon } \\
\text { vs. filcon II } 3\end{array}$ & $\begin{array}{l}\text { delefilcon vs. } \\
\text { filcon II } 3\end{array}$ & $\begin{array}{l}\text { delefilcon vs. } \\
\text { narafilcon }\end{array}$ & $\begin{array}{l}\text { narafilcon vs. } \\
\text { filcon II } 3\end{array}$ & $\begin{array}{l}\text { delefilcon vs. } \\
\text { filcon II } 3\end{array}$ & $\begin{array}{l}\text { delefilcon vs. } \\
\text { narafilcon }\end{array}$ & $\begin{array}{l}\text { narafilcon } \\
\text { vs. filcon II } 3\end{array}$ & $\begin{array}{c}\text { delefilcon vs. } \\
\text { filcon II } 3\end{array}$ \\
\hline 8 hours & $0.527^{*}$ & 0.169 & 0.280 & 0.356 & 0.161 & 0.057 & -0.116 & 0.175 & -0.156 \\
\hline 12 hours & 0.151 & 0.214 & -0.057 & 0.335 & $0.626^{* *}$ & 0.416 & 0.201 & -0.137 & -0.073 \\
\hline 16 hours & 0.155 & 0.080 & 0.386 & 0.263 & 0.257 & 0.356 & 0.004 & 0.064 & -0.0143 \\
\hline Brand & $8 v s 12$ & $8 v s 16$ & $12 v s 16$ & $8 v s 12$ & $8 v s 16$ & $12 v s 16$ & $8 v s 12$ & $8 v s 16$ & $12 v s 16$ \\
\hline narafilcon A & 0.318 & 0.317 & 0.355 & $0.525^{* *}$ & $0.438^{*}$ & $0.633^{* *}$ & 0.372 & -0.306 & -0.122 \\
\hline delefilcon A & 0.284 & 0.345 & 0.194 & $0.494^{*}$ & $0.529 * *$ & $0.660 * *$ & 0.038 & 0.219 & -0.009 \\
\hline filcon II 3 & -0.004 & 0.200 & 0.223 & 0.303 & $0.499 *$ & $0.562 * *$ & 0.311 & 0.051 & 0.061 \\
\hline
\end{tabular}

$*=p<0.01 ; * *=p<0.001$ 


\section{Ocular Comfort}

Lens fit was generally not correlated with subjective comfort, but comfort varied little between subjects in this young healthy population (Table 3). Having better comfort than other subjects with one lens brand did not result in having better comfort with other brands (Table 3). The change in lens fit (movement on blink, lag and push-up) between 8 and 12 hours of wear and between 8 and 16 hours of lens wear also did not correlate with the change in comfort over these times for any of the lenses tested (Spearman's Rank correlation $p>0.05$; Table 3). When wearing each lens brand, the rating of comfort correlated between 8,12 and 16 hour of wear assessments (Table 4).

\section{Ocular Physiology}

Lens fit was generally not correlated with bulbar or limbal hyperaemia (Table 5). However, having less pronounced bulbar hyperaemia than other subjects with one lens brand resulted in less bulbar hyperaemia with other brands, although this was not the case after 16 hours of wear (Table 5). There was an association between limbal hyperaemia when wearing delefilcon A lenses and other brands. When wearing each lens brand, the grading of bulbar and limbal hyperaemia generally correlated between 8,12 and 16 hour of wear assessments (Table 4). Lens fit was not correlated with end of day (16 hour) corneal or conjunctival staining (Table 5). Having more corneal or conjunctival staining than other subjects with one lens brand did not result in having a high level of staining with other brands (Table 5).

\section{Lens Wettability}

Lens fit was generally not correlated with non-invasive lens surface break-up time or tear meniscus height (Table 6). Having a more wettable contact lens surface than other subjects with one lens brand was not generally associated with a higher surface wettability with the other brands (Table 6). When wearing each lens brand, the grading of non-invasive lens surface tear break-up time was correlated between 12 and 16 hour of wear and for the narafilcon additionally between 8 and 16 hours of lens wear and tear meniscus height between 12 and 16 hours (Table 4). 
Table 3: Effect of lens fit (correlation coefficients) on comfort and relationship between lens brands $\quad$ (n=39) $* P<0.01$; $* * P<0.001$

\begin{tabular}{|c|c|c|c|c|c|c|c|c|c|c|c|c|}
\hline \multirow[b]{2}{*}{ Time } & \multicolumn{3}{|c|}{ narafilcon A } & \multicolumn{3}{|c|}{ delefilcon A } & \multicolumn{3}{|c|}{ filcon II 3} & \multicolumn{3}{|c|}{ Comfort between Brands } \\
\hline & Blink & Lag & Push-up & Blink & Lag & Push-up & Blink & Lag & Push-up & $\begin{array}{l}\text { delefilcon vs } \\
\text { narafilcon }\end{array}$ & $\begin{array}{c}\text { narafilcon } \\
\text { vs filcon II } 3\end{array}$ & $\begin{array}{c}\text { delefilcon vs } \\
\text { filcon II } 3\end{array}$ \\
\hline 8 hours & 0.119 & -0.346 & 0.106 & -0.091 & -0.11 & 0.148 & 0.244 & -0.012 & 0.250 & -0.047 & 0.048 & 0.029 \\
\hline 12 hours & 0.121 & -0.104 & 0.066 & -0.130 & -0.11 & -0.020 & 0.163 & -0.095 & 0.232 & 0.014 & 0.089 & 0.094 \\
\hline 16 hours & -0.060 & 0.127 & 0.217 & -0.163 & -0.14 & 0.253 & 0.032 & 0.051 & 0.027 & 0.262 & 0.390 & -0.059 \\
\hline
\end{tabular}

Table 4: Correlation between difference time points for ocular comfort, physiology and lens wettability witheach lens brand $\quad(\mathrm{n}=39$ ) $* \boldsymbol{P}<0.01 ; * * \boldsymbol{P}$ $<0.001$

\begin{tabular}{|c|c|c|c|c|c|c|c|c|c|c|c|c|c|c|c|}
\hline & \multicolumn{3}{|c|}{ Ocular Comfort } & \multicolumn{3}{|c|}{ Bulbar Hyperaemia } & \multicolumn{3}{|c|}{ Limbal Hyperaemia } & \multicolumn{3}{|c|}{ NITBUT } & \multicolumn{3}{|c|}{ ТМH } \\
\hline & 8vs12 & $8 v s 16$ & $12 v s 16$ & $8 v s 12$ & $8 v s 16$ & $12 v s 16$ & $8 v s 12$ & $8 v s 16$ & $12 v s 16$ & 8vs12 & $8 v s 16$ & $12 v s 16$ & $8 v s 12$ & $8 v s 16$ & $12 v s 16$ \\
\hline narafilcon A & $0.846 * *$ & $0.650 * *$ & $0.704 * *$ & $0.501^{*}$ & 0.092 & 0.292 & $0.493^{*}$ & $0.611 * *$ & $0.594^{* *}$ & 0.128 & $0.498^{*}$ & $0.449 *$ & 0.123 & 0.128 & $0.498^{*}$ \\
\hline delefilcon A & $0.515^{* *}$ & $0.645^{* *}$ & $0.657^{* *}$ & $0.453^{*}$ & $0.577^{* *}$ & $0.459 *$ & $0.621^{* *}$ & $0.668^{* *}$ & $0.554 *$ & 0.347 & 0.283 & $0.553 * *$ & 0.337 & 0.347 & 0.283 \\
\hline filcon II 3 & $0.684 * *$ & $0.484 *$ & $0.594 * *$ & $0.587^{* *}$ & $0.576 * *$ & $0.559 * *$ & $0.512 *$ & $0.532 * *$ & $0.616 * *$ & 0.228 & 0.424 & $0.756 * *$ & 0.387 & 0.228 & 0.424 \\
\hline
\end{tabular}


Table 5: Effect of lens fit (correlation coefficients) on ocular physiology and relationship between lens brands $\quad(\mathrm{n}=39) \quad * P<0.01 ; * * P<0.001$

\begin{tabular}{|c|c|c|c|c|c|c|c|c|c|c|c|c|}
\hline \multirow[b]{2}{*}{ Time } & \multicolumn{3}{|c|}{ narafilcon $A$} & \multicolumn{3}{|c|}{ delefilcon A } & \multicolumn{3}{|c|}{ filcon II 3} & \multicolumn{3}{|c|}{ Between Brands } \\
\hline & Blink & Lag & Push-up & Blink & Lag & Push-up & Blink & Lag & Push-up & $\begin{array}{l}\text { delefilcon vs. } \\
\text { narafilcon }\end{array}$ & $\begin{array}{l}\text { narafilcon vs. } \\
\text { filcon // } 3\end{array}$ & $\begin{array}{l}\text { delefilcon vs. } \\
\text { filcon II } 3\end{array}$ \\
\hline \multicolumn{13}{|c|}{ Bulbar Hyperaemia } \\
\hline 8 hours & -0.036 & -0.093 & -0.164 & 0.306 & 0.071 & 0.076 & 0.029 & -0.276 & -0.039 & $0.663^{*}$ & $0.527 * *$ & $0.496 *$ \\
\hline 12 hours & 0.196 & -0.092 & 0.146 & 0.128 & -0.211 & 0.146 & -0.149 & -0.370 & 0.144 & $0.504^{*}$ & 0.297 & $0.397^{*}$ \\
\hline 16 hours & 0.234 & -0.113 & 0.198 & 0.283 & 0.001 & 0.226 & -0.006 & -0.276 & 0.378 & 0.123 & 0.211 & 0.389 \\
\hline \multicolumn{13}{|c|}{ Limbal Hyperaemia } \\
\hline 8 hours & -0.024 & -0.369 & -0.131 & 0.249 & 0.356 & -0.055 & 0.176 & 0.065 & -0.100 & 0.377 & 0.266 & 0.440 \\
\hline 12 hours & 0.228 & 0.078 & 0.086 & 0.080 & -0.084 & 0.124 & 0.001 & -0.033 & 0.093 & $0.589 * *$ & 0.285 & $0.542^{* *}$ \\
\hline 16 hours & 0.199 & 0.081 & 0.072 & 0.259 & -0.032 & 0.262 & -0.206 & -0.188 & 0.180 & $0.482^{*}$ & 0.275 & 0.230 \\
\hline \multicolumn{13}{|c|}{ Corneal Staining } \\
\hline 16 hours & 0.216 & -0.013 & -0.178 & -0.172 & -0.198 & -0.111 & -0.123 & -0.109 & -0.105 & 0.057 & 0.196 & 0.303 \\
\hline \multicolumn{13}{|c|}{ Conjunctival Staining } \\
\hline 16 hours & 0.358 & -0.089 & -0.216 & 0.152 & 0.037 & 0.134 & -0.147 & 0.078 & 0.106 & 0.330 & 0.154 & 0.027 \\
\hline
\end{tabular}


Table 6: Effect of lens fit (correlation coefficients) on ocular physiology and relationship between lens brands $(\mathrm{n}=39) \quad * P<0.01 ; * * P<0.001$

\begin{tabular}{|c|c|c|c|c|c|c|c|c|c|c|c|c|}
\hline \multirow[b]{2}{*}{ Time } & \multicolumn{3}{|c|}{ narafilcon $A$} & \multicolumn{3}{|c|}{ delefilcon A } & \multicolumn{3}{|c|}{ filcon II 3} & \multicolumn{3}{|c|}{ Between Brands } \\
\hline & Blink & Lag & Push-up & Blink & Blink & Lag & Push-up & Blink & Blink & $\begin{array}{c}\text { delefilcon vs } \\
\text { narafilcon }\end{array}$ & $\begin{array}{l}\text { narafilcon vs } \\
\text { filcon II } 3\end{array}$ & $\begin{array}{l}\text { delefilcon vs } \\
\text { filcon II } 3\end{array}$ \\
\hline \multicolumn{13}{|c|}{ Non-Invasive Lens Surface Tear Break-Up Time } \\
\hline 8 hours & -0.135 & 0.164 & 0.098 & 0.217 & 0.022 & 0.077 & 0.041 & 0.182 & 0.369 & -0.115 & 0.125 & 0.083 \\
\hline 12 hours & -0.004 & -0.279 & -0.191 & 0.182 & -0.174 & 0.016 & -0.140 & -0.359 & 0.108 & 0.035 & 0.235 & 0.098 \\
\hline 16 hours & 0.053 & 0.039 & -0.048 & 0.086 & 0.012 & 0.297 & 0.195 & -0.157 & 0.163 & $0.524 * *$ & 0.405 & 0.109 \\
\hline \multicolumn{13}{|c|}{ Lens Surface Tear Meniscus Height } \\
\hline 8 hours & 0.073 & -0.053 & -0.183 & -0.169 & 0.038 & 0.117 & -0.123 & -0.062 & -0.125 & -0.234 & 0.295 & 0.025 \\
\hline 12 hours & -0.084 & 0.260 & 0.095 & -0.411 & 0.069 & -0.082 & -0.024 & 0.012 & 0.031 & 0.289 & $0.458^{*}$ & -0.016 \\
\hline 16 hours & -0.254 & -0.011 & -0.172 & -0.090 & -0.153 & 0.283 & -0.146 & -0.048 & -0.103 & $0.415^{*}$ & 0027 & -0.028 \\
\hline
\end{tabular}




\section{DISCUSSION}

This study shows lens fit changes between 8 hours, the longest duration of wear previously examined, and 16 hours, which encompasses the wearing day of the majority of contact lens wearers. While one might intuitively expect all lens mobility metrics to change in the same direction, movement on blink remained relatively constant over this period, the lens lag on horizontal excursion decreased by approximately $10 \%$, but conversely the push-up recovery speed increased by about $20 \%$.

On excursion, the anatomical interaction between the eyelid and ocular surface displaces the lens, which results in lens lag. This interaction is unlikely to change during the day so it can be presumed the friction between the lens surfaces and the ocular anatomy which is associated with the tear film changes over the day results in the decrease seen between 8 and 16 hours of wear. However, lens fit including lag was generally not correlated with measures of lens surface wettability, in the form of non-invasive lens surface break-up time or tear meniscus height. These measures seem reasonably robust for each lens brand investigated, with the measures being correlated between some of the time points. However, these tear film metrics only assess anterior surface lens wettability and hence the key frictional component that changes towards the end of the day may be between the rear surface of the lens and the ocular surface due to potential changes in tear composition (although this has not been researched in the peer reviewed literature) or the effect of tear composition on the lens curvature due to hydration. ${ }^{18}$ The lack of change in movement with blink over this period may result from this increase in back surface friction having a greater effect on the horizontal meridian (the direction that lag was assessed), than the steeper vertical meridian (the direction movement on blink was assessed) in this young population of principally with-the-rule low astigmats.

Some authors have investigated the ease of push-up rather than just recovery speed, which would be related to lens binding as well as friction between the ocular and posterior lens surfaces. However, push-up recovery speed has been shown to independently contribute to overall lens mobility, unlike ease of push-up, and can be 
assessed in-vivo objectively. ${ }^{16}$ The push-up recovery speed involves greater displacement of the lens than assessment of lag, as well as being influenced by the friction between the ocular and posterior lens surface, it is also moderated by the elastic properties of the lens. Traditional lenses are known to dehydrate towards the end of the day, ${ }^{18}$ increasing its modulus, and hence it could be postulated that this results in the increase in push-up recovery speed with time.

Objective assessment of lens fit was not strongly correlated between lens brands despite their similar base curves and diameters. Previous studies have shown that changing the base curve of a soft lens does not generally have a significant effect on lens fit $^{16}$ and this is because the lens fit is influenced by peripheral corneal topography ${ }^{19}$ and the corneoscleral anatomy. ${ }^{20}$ Currently marketed daily disposable silicone-hydrogel contact lenses differ in shape profile and material composition and the lack of correlation in lens fit between the brands indicates these features affect the lens fit differently for individual patients. Hence clinically, if a trialled lens does not fit adequately, it is appropriate to trial fit another brand of lens even if the stated base curve and diameter parameters are similar. It also proves beyond doubt that lens substitution, even of a lens with similar base curve and diameter parameters, without a clinical lens assessment, is inappropriate.

Lag was the fit characteristic that was best correlated between assessment times with each individual lens brand. Hence lens mobility in the vertical meridian as assessed by movement on blink or push-up recovery speed, may be a less reliable indicator of lens fit than lag when investigating differences between lens brands. Subjective assessment of ocular comfort was consistent between assessment times with each lens brand (i.e. subjects with the best comfort within the cohort at one time point were likely to have the best comfort at subsequent time points and vice versa, even if the overall comfort had reduced), suggesting it is a robust measure. Lens fit was generally not correlated with subjective comfort over the end of a day. It is possible that a population with less comfortable eyes may have been more sensitive to changes in lens fit. Having better comfort than other subjects with one lens brand did not result in having better comfort 
with other brands. Therefore it is the lens-patient interaction that drives lens comfort, rather than the lens design/material or patient in isolation. Hence clinically, if a patient is uncomfortable in their current lenses, it does not necessarily follow that they will be uncomfortable in another brand.

Grading of bulbar and limbal hyperaemia was consistent between assessment times with each lens brand, suggesting they are also robust measures. Lens fit was generally not correlated with bulbar or limbal hyperaemia. However patients who exhibit greater redness with one lens brand often had a greater redness with the other brands and vice versa. Limbal hyperaemia is associated with ocular insult, ${ }^{21}$ however, in this study there was no difference in lens fit or ocular staining between the investigated lenses. Limbal hyperaemia is also associated with insufficient oxygen transmissibility, ${ }^{22}$ so the correlation between delefilcon A lenses and the other brands, but not between narafilcon A and filcon II 3, may result from the higher oxygen transmissibility difference inherent between the former, even in daily wear. The lack of relationship in corneal or conjunctival staining between brands on lens removal suggests that staining is not related just to patient susceptibility, but an interaction between an individual and a particular lens design. $^{23}$

In-vivo lens surface wettability was a less consistent measure across time for a particular brand than subjective comfort and ocular physiology. Clinical tear film techniques have previously been shown to be susceptible to wide variation between repeated measurement and this may contribute to this finding ${ }^{24}$ Non-invasive lens surface breakup time or tear meniscus height was not affected by lens fit in initially acceptable fitting lenses as expected. However, having a more wettable contact lens surface with one brand was not associated with having a more wettable surface with another brand, suggesting that lens surface wetting is not related just to lens characteristics, but an interaction between an individual's ocular surface including their tear film and interaction with the lens. 
In conclusion, this study shows objectively that lens fit changes between 8 hours and the end of the typical contact lens wearing day. However, lens fit in initially acceptable fitting lenses it is not associated with ocular comfort, ocular physiology or lens surface wettability. If a lens fits adequately or is comfortable, it does not follow that another lens brand of similar base curve and diameter parameters will fit acceptably and/or be comfortable, so lens substitution, without a clinical lens assessment, is unadvisable.

\section{Acknowledgement}

The study was funded by Alcon Laboratories. 


\section{REFERENCES:}

1. Riley C, Young G, Chalmers R. Prevalence of ocular surface symptoms, signs, and uncomfortable hours of wear in contact lens wearers: the effect of refitting with daily-wear silicone hydrogel lenses (senofilcon a). Eye Contact Lens 2006;32:281-6.

2. Begley CG, Chalmers RL, Mitchell GL, Nichols KK, Caffery B, Simpson T, DuToit R, Portello J, Davis L. Characterization of ocular surface symptoms from optometric practices in North America. Cornea. 2001;20:610-8.

3. Long B, McNally J. The clinical performance of a silicone hydrogel lens for daily wear in an Asian population. Eye Contact Lens 2006;32:65-71.

4. Maruyama K, Yokoi N, Takamata A, Kinoshita S. Effect of environmental conditions on tear dynamics in soft contact lens wearers. Investig Ophthalmol Vis Sci 2004;45:2563-8.

5. Peterson RC, Wolffsohn JS, Nick J, Winterton L, Lally J. Clinical performance of daily disposable soft contact lenses using sustained release technology. Contact Lens Anterior Eye 2006;29:127-34.

6. Wolffsohn JS, Hunt OA, Chowdhury A. Objective clinical performance of 'comfort-enhanced' daily disposable soft contact lenses. Contact Lens Anterior Eye 2010;33:88-92.

7. Riley C, Chalmers RL, Pence N. The impact of lens choice in the relief of contact lens related symptoms and ocular surface findings. Contact Lens Anterior Eye 2005;28:13-9.

8. Pritchard N, Fonn D, Brazeau D. Discontinuation of contact lens wear: a survey. Int Cont Lens Clin 1999;26:157-62.

9. Santodomingo-Rubido J, Barrado-Navascues E, Rubido-Crespo MJ. Ocular surface comfort during the day assessed by instant reporting in different types of contact and non-contact lens wearers. Eye Contact Lens 2010;36:96-100.

10. Fonn D, Dumbleton K. Dryness and discomfort with silicone hydrogel contact lenses. Eye Contact Lens 2003;29:S101-4; discussion S15-8, S92-4.

11. Papas EB, Tilia D, Tomlinson D, Williams J, Chan E, Chan J, Golebiowski B. Consequences of wear interruption for discomfort with contact lenses. Optom Vis Sci. 2014;91:24-31.

12. Willcox M, Phillips B, Ozkan J, Jalbert I, Meagher L, Gengenbach T, Holden B, Papas E. Interactions of lens care and silicone hydrogel lenses and effect on comfort. Optom Vis Sci 2010;87.

13. Keir N, Woods CA, Dumbleton K, Jones L. Clinical performance of different care systems with silicone hydrogel contact lenses. Contact Lens Anterior Eye 2010;33:189-95.

14. Dumbleton K, Woods C, Jones L, Richter D, Fonn D. Comfort and vision with silicone hydrogel lenses: effect of compliance. Optom Vis Sci. 2010;87:421-5.

15. Sorbara L, Peterson R, Woods C, Fonn D. Multipurpose disinfecting solutions and their interactions with a silicone hydrogel lens. Eye Contact Lens 2009;35:92-7.

16.. Wolffsohn JS, Hunt OA, Basra AK. Simplified recording of soft contact lens fit. Contact Lens Anterior Eye 2009;32:37-42.

17. Wolffsohn JS, Purslow C. Clinical monitoring of ocular physiology using digital image analysis. Contact Lens Anterior Eye 2003;26:27-35.

18. Tranoudis I, Efron N. Parameter stability of soft contact lenses made from different materials. Contact Lens Anterior Eye 2004;27:115-31.

19. Hall LA, Young G, Wolffsohn JS, Riley C. The influence of corneoscleral topography on soft contact lens fit. Invest Ophthalmol Vis Sci 2011;52:6801-6.

20. Hall LA, Hunt C, Young G, Wolffsohn J. Factors affecting corneoscleral topography. Invest Ophthalmol Vis Sci 2013;54:3691-701.

21. Young G, Coleman S. Poorly fitting soft lenses affect ocular integrity. CLAO J 2001;27:68-74.

22. Papas E. On the relationship between soft contact lens oxygen transmissibility and induced limbal hyperaemia. Exp Eye Res 1998;67:125-31.

23. Maissa C, Guillon M, Garofalo RJ. Contact lens-induced circumlimbal staining in silicone hydrogel contact lenses worn on a daily wear basis. Eye Contact Lens 2012;38:16-26.

24. Best N, Drury L, Wolffsohn JS. Clinical evaluation of the Oculus Keratograph. Contact Lens Anterior Eye 2012;35:171-4. 